\title{
Geroscience and the Role of Aging in the Etiology and Management of Alzheimer's Disease
}

\author{
F. Sierra \\ Correspondance author: Division of Aging Biology, National Institute on Aging (NIA), National Institutes of Health (NIH) - 7201 Wisconsin Ave, Suite 3N300, Bethesda MD \\ 20892, USA, sierraf@nia.nih.gov
}

Key words: Geroscience, aging, Alzheimer's, neurodegeneration.

J Prev Alz Dis 2020;1(7):2-3

Published online December 5, 2019, http:/ / dx.doi.org/10.14283/jpad.2019.49

W ith the relentless aging of the population worldwide, two major concerns need our immediate attention: the expected dramatic increase in disease and disability burden, and the decrease in the ratio of working individuals vs. retirees. A comprehensive approach involving experts in many disciplines will be required to tackle these issues. Here we will concentrate on the role of basic biological science in averting the increase in disease and disability, using Alzheimer's Disease (AD) as a model.

The spectrum of $\mathrm{AD}$ represents a serious threat and a psychological burden on people at all ages. The US Alzheimer's Association estimates that 5.8 million Americans are currently living with Alzheimer's, and 1 out of 3 seniors dies with the disease or other dementias. Because of its insidious effect on both the individual and his/her surroundings, as well as the associated healthcare cost, AD has been singled out for special efforts by funding agencies and scientists alike, and while some progress has been made, it is clearly not sufficient. Indeed, in the past few decades, scientists have been able to identify the molecular composition of the telltale plaques and tangles and have created a large number of mouse models that, to some extent, recapitulate the pathological characteristics of the disease (1). Furthermore, early efforts identified some major drivers of the familial (rare) form of the disease, and more recently, a large number of genes suspected to play a role on the late-onset, non-familial form of the disease have also been identified (2). The role of these new genes is just beginning to be unraveled. Yet a cure has been elusive, and even prevention strategies have been less than hoped for.

One aspect of the etiology of the disease that has been largely neglected until recent years is the role of aging. It is not by mere chance that so many chronic diseases appear simultaneously, in many species, as individuals reach approximately $2 / 3$ of the lifespan for their species (around 60 years for humans). Most of these chronic diseases differ dramatically from the diseases we were able to conquer in the 20th century, in that they are not caused by external agents such as pathogens and poor environmental quality but rather, they are the result of failures within our own organism. For that reason, these diseases have proven to be less tractable, and fighting them is more complex. But the age-dependency in the appearance of symptoms from multiple chronic diseases belies the fact that aging is by far the major risk factor for most of these chronic conditions (3), including Alzheimer's disease (4). Importantly, the fact that such diseases occur in multiple species at different chronological times (days in flies, months in mice, years in humans), but always at the same physiological time (about $2 / 3$ of the expected lifespan) indicates that it is the process of aging, not the passage of time, that is central. The passage of time indeed allows the accumulation of damage that can lead to disease and disability. However, this accumulation is often rather slow while the organism is young and resilient. It is only after the process of aging starts weakening that resilience that serious accumulation of damage - and thus disease - occurs. So, it is not simply that as we age, damage has accumulated to an extent that causes disease; rather, it is that as we age, we had lost part of our defenses, thus allowing the damage to accumulate. Taking AD as an example, we know that even individuals with the worst genetic predisposition to the disease won't develop symptoms when they are toddlers or teenagers, they will develop them late in life (earlier than other, non-genetically afflicted populations, but usually not earlier than their 40 s or 50 s) (5). Yet, because of their genetic burden, they are producing enormous amounts of deleterious aggregation-prone proteins from before birth! Minimal accumulation and no disease occurs because, while young, their resilience capacity allows them to counteract this burden, and resolve much of the damage through proteostasis mechanisms.

This is not unique to $\mathrm{AD}$, and a similar argument can be brought to bear in many other chronic diseases of the elderly, including cancer, cardiovascular, chronic kidney disease, etc. This is the central tenet of the new field of geroscience: since aging is at the core, and the 
most important risk factor for so many chronic diseases and conditions, it follows that addressing aging will produce a better outcome than addressing each disease individually (6). Indeed, it is expected that, by slowing down the pace of aging we can delay all such chronic ailments, all at the same time. This is nothing new, since we have always known about the fragility and illnesses that often accompany old age. What is new is the amazing advancements we have had in the last couple of decades in our efforts to understand the biological underpinnings of the aging process. Indeed, scientists have now identified a handful of molecular and cellular pathways that drive the process of aging $(7,8)$. Moreover, those discoveries have led to the identification of pharmacological and dietary means to slow down aging processes, and some of these interventions are already being tried in the clinic, including rapamycin (9) and senolytics (10).

The AD field has been slow to recognize these developments, but changes are being implemented, among others, through several new initiatives promoted by the US National Institute of Aging, aimed at promoting research into the geroscience underpinnings of AD. In fact, pre-clinical data in various mouse models of AD suggest that interventions aimed at slowing down the aging process might be effective in delaying or slowing down disease progression. As in other diseases that affect preferentially the elderly population, these pre-clinical interventions have focused primarily on rapamycin $(11,12)$ and senolytics (13). In fact, a strong argument has been made to test rapamycin in clinical trials of the disease (14), and two small phase I trials of senolytics are being planned for the near future (J. Kirkland, pers. comm.). In the accompanying paper by Guerville et al., a vigorous argument is made for the inclusion of geroscience principles in our fight to conquer Alzheimer's disease. Importantly, the paper also outlines specific areas where attention to the pillars of aging might be fruitful in our efforts against Alzheimer's.

Disclosures: Dr. Sierra has no conflicts to disclose. The ideas discussed represent Dr. Sierra's views and do not represent the views of the U.S. government.

\section{References}

1. Hardy J. A hundred years of Alzheimer's disease research. Neuron. 2006;52:313.

2. Naj A.C., Schellenberg G.D. and the Alzheimer's Disease Genetics Consortium (ADGC). Genomic variants, genes, and pathways of Alzheimer's disease: An overview. Am J Med Genet B Neuropsychiatr Genet. 2017;174:5-26.

3. Sierra F. and Kohanski R. Geroscience and the trans-NIH Geroscience Interest Group, GSIG. Geroscience 39:1-5.

4. Qiu C., Kivipelto M. and Strauss E. Epidemiology of Alzheimer's disease: Occurrence, determinants and strategies toward intervention. Dialogues Clin. Neurosci. 2009;11:111-128.

5. Ryman D.C., Acosta-Baena N., Aisen P.S. et al. Symptom onset in autosomal dominant Alzheimer's disease: a systematic review and meta-analysis. Neurology 2014;83:253-260.

6. Austad SN. The geroscience hypothesis: Is it possible to change the rate of aging? in Advances in Geroscience, Sierra \& Kohanski eds., Springer, 2016;pp1-36.

7. López-Otín C., Blasco M.A., Partridge L. et al. The hallmarks of aging. Cell 2013;153:1194-217.

8. Kennedy B.K., Berger S, Brunet A. et al. Geroscience: linking aging to chronic disease. Cell 2014;159:709-713.

9. Mannick JB, Del Giudice G, Lattanzi M et al. mTOR inhibition improves immune function in the elderly. Sci Transl Med. 2014;6:268ra179

10. Justice JN, Nambiar AM, Tchkonia T et al. Senolytics in idiopathic pulmonary fibrosis: Results from a first-in-human, open-label, pilot study. EBioMedicine. 2019;40:554-563.

11. Caccamo A., Majumder S., Richardson A. et al. Molecular interplay between mammalian target of rapamycin (mTOR), amyloid- $\beta$ and tau: Effects on cognitive impairments. J. Biol. Chem. 2010;285:13107-13120.

12. Lin A-L., Jahrling J.B., Zhang W. et al. Rapamycin rescues vascular, metabolic and learning deficits in apolipoprotein E4 transgenic mice with presymptomatic Alzheimer's disease. J. Cereb. Blood Flow Metab. 2017;37:217226.

13. Bussian T.J., Aziz A., Meyer C.F. et al. Clearance of senescent glial cells prevents tau-dependent pathology and cognitive decline. Nature 2018;562:578-582.

14. Kaeberlein M. and Galvan V. Rapamycin and Alzheimer's disease: time for a clinical trial? Sci. Transl. Med. 2019;11, eaar4289 